\section{AB0201 \\ IMPACT OF TREATMENT INITIATION DELAY ON DISEASE ACTIVITY DURING RHEUMATOID ARTHRITIS}

H. Bettaieb ${ }^{1}$, A. Fazaa ${ }^{1}$, S. Miladi ${ }^{1}$, M. Sellami ${ }^{1}$, O. Kmar ${ }^{1}$, L. Souabni ${ }^{1}$, S. Kassab ${ }^{1}$, S. Chikilii ${ }^{1}$, Z. Leith ${ }^{1}$, K. Ben Abdelghani ${ }^{1}$, A. Laatar ${ }^{1} .{ }^{1}$ Mongi Slim Hospital, Tunis, Tunisia

Background: During rheumatoid arthritis (RA), initiating conventional synthetic Disease Modifying Anti-Rheumatic Drug (csDMARD) at the early stages of the disease is a mandatory condition to achieve DMARD-free sustained remission (1). Limited data studying the relationship between RA treatment delay and disease activity are available.

Objectives: The aim of this study was to assess the impact of csDMARD initiation delay during RA on disease activity.

Methods: This is a cross-sectional study including patients with RA (ACR/ EULAR criteria).Delays were collected from patients' interview and were represented respectively by D1, D2 and D3. D1 stands for the lag time separating the first RA symptom onset and rheumatologist consultation. D2 stands for the lag time separating the first RA symptom onset and RA diagnosis. D3 stands for lag time separating the first RA symptom onset and csDMARD initiation. Disease activity was evaluated by: Visual Analogue Scale for pain (VAS), number of tender joints, number of swollen joints, erythrocyte sedimentation rate (ESR), C-reactive protein (CRP) and Disease Activity Score28 (DAS28). The data were analyzed with descriptive statistics, Student's t test, chi (2) test, and Spearman correlation using the SPSS statistical package. A p value $<0.05$ was considered significant.

Results: The study included 100 RA patients ( 86 women and 14 men), with a mean age of $56.5 \pm 12.4$ years. The mean age at the onset of RA was $47.5 \pm 12.4$ years. Median D1, D2 and D3 were respectively 12 months [0-242], 15.7 months [2-252] and 18 months [2-270]. Methotrextate was prescribed in $86 \%$ of cases. At RA diagnosis, the median values for the following parameters were: VAS 80 [30-100], number of tender joints 10[0-28], number of swollen joints 5 [0-17], ESR 43mm/hour [6-133], CRP $14.1 \mathrm{mg} /$ [ [1-120], DAS28 (ESR) 5.22 [2-7.52] and DAS28 (CRP) 4.6 [1-6.93]. After one year of follow-up, the median parameters of the disease activity were respectively: VAS 60 [0-100], number of tender joints 6[0-28], number of swollen joints 2 [0-22], ESR $32 \mathrm{~mm} /$ hour [2-106], CRP $7.5 \mathrm{mg} / \mathrm{l}$ [1.2-94], DAS28 (ESR) 4.1 [1.4-7.1] and DAS28 (CRP) 3.7 [1.68-6.22]. Significant positive correlation was found between delays in csDMARD initiation and DAS28 (CRP) scores over the first year $(\mathrm{p}=0.02, r=0.29)$.

Conclusion: In this study, delays in treatment were associated with higher DAS28 (CRP) scores after one year of follow-up. Our results suggest that early identification and treatment of RA leads to improved outcomes and even improved rates of drug-free remission.

References:

[1] Van Nies JA, Krabben A, Schoones JW, et al. What is the evidence for the presence of a therapeutic window of opportunity in rheumatoid arthritis? A systematic literature review. Ann Rheum Dis 2014;73:861-70.

Disclosure of Interests: None declared

DOI: 10.1136/annrheumdis-2020-eular.6144

\section{AB0202 GENETIC SUSCEPTIBILITY AND PHENOTYPE OF RHEUMATOID ARTHRITIS IN DANISH AND TURKISH PATIENTS}

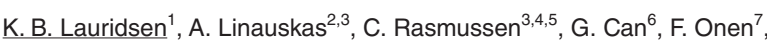
L. Dreyer ${ }^{8,9}$, R. Steffensen ${ }^{1}$, K. R. Nielsen ${ }^{1}$, N. Steen Krogh ${ }^{10,11}$, S. Akar ${ }^{12,13}$, N. Akkoc ${ }^{14} .{ }^{1}$ Aalborg University Hospital, Clinical Immunology, Aalborg, Denmark; ${ }^{2}$ North Denmark Rerional Hospital, Hjorring, Denmark; ${ }^{3}$ DANBIO, Hjorring, Denmark; ${ }^{4}$ North Denmark Regional Hospital., Rheumatology, Hjorring, Denmark; Institute of Clinical Medicine, Aalborg University, Denmark, Aalborg, Denmark; ${ }^{6}$ Dokuz Eylul University, Faculty of Medicine, Internal Medicine, Division of Rheumatology, Izmir, Turkey; ${ }^{7}$ Dokuz Eylul University School of Medicine, Internal Medicin, Division of Rheumatology, Izmir, Turkey; ${ }^{8}$ Aalborg University Hospital, Rheumatology, Aalborg, Denmark; ${ }^{9}$ Institute of Clinical Medicine, Aalborg University, Aalborg, Denmark; ${ }^{10}$ Zitelab, Copenhagen, Denmark; ${ }^{11}$ DANBIO, Copenhagen, Denmark; ${ }^{12}$ Izmir Ataturk Education and Research Hospital, Internal Medicine Clinic, Izmir, Turkey; ${ }^{13}$ Izmir Katip Celebi University School of Medicine, Internal Medicine, Division of Rheumatology, Izmir, Turkey; ${ }^{14}$ Celal Bayar University, Internal Medicine, Division of Rheumatology, Manisa, Turkey

Background: Previous studies indicate that genetic susceptibility and phenotype of rheumatoid arthritis (RA) differ between the populations.

Objectives: To compare the clinical, serologic expression and the presence of shared epitopes (SE) of incident RA in two different populations, one from Northern and the other from Southern Europe.

Methods: Data on incident RA patients fulfilling EULAR/ACR 2010 classifications criteria for RA were collected at Rheumatology Departments in Denmark and Turkey in 2015-2016. Patients were assessed using the same standardized protocol in both populations. SE carrier status were assigned, according to the du Montcel classification based, into six allele groups: S1, S2, S3D, S3P and $X$, where S2 and S3P are RA risk-enhancing alleles and S1 and S3D are RA protective alleles of the shared epitope(1).

Results: 109 incident RA patients from Denmark and 114 incident RA patients from Turkey were enrolled. Genetic data were available from $87 \%$ of the patients.

Table 1. Characteristics of incident rheumatoid arthritis patients in Denmark and Turkey

\begin{tabular}{lccc}
\hline & $\begin{array}{c}\text { Danish patients } \\
\mathrm{n}=109\end{array}$ & $\begin{array}{c}\text { Turkish patients } \\
\mathrm{n}=114\end{array}$ & P-value \\
\hline Age at diagnosis, years & $60(49-69)$ & $52(43-64)$ & 0.003 \\
Female, \% & 64 & 74 & 0.12 \\
Symptom duration, months & $7(4-21)$ & $6(2-22)$ & 0.6 \\
Smoking status & & & \\
$\quad$ Never smoker, \% & 43 & 44 & 0.98 \\
$\quad$ Former smoker, \% & 28 & 31 & 0.68 \\
$\quad$ Current smoker, \% & 29 & 25 & 0.54 \\
VAS pain (0-100mm) & $45(28-66)$ & $60(41-72)$ & 0.01 \\
VAS fatigue (0-100mm) & $51(29-69)$ & $50(20-70)$ & 0.32 \\
VAS global, patient (0-100 mm) & $60(31-80)$ & $60(41-73)$ & 0.77 \\
Swollen joint count (0-28) & $7(4-11)$ & $3(1-6)$ & $<0.00001$ \\
Tender joint count (0-28) & $7(3-11)$ & $5(2-8)$ & 0.04 \\
HAQ score (0-3) & $0.75(0.34-1.25)$ & $1.0(0.25-1.75)$ & 0.02 \\
DAS28 & $4.7(4.1-5.5)$ & $4.3(3.3-5.2)$ & 0.01 \\
CRP, mg/l & $7(3.0-18.5)$ & $8(3.1-22.6)$ & 0.54 \\
IgM RF positive, \% & 70 & 66 & 0.58 \\
ACPA positive, \% & 63 & 75 & 0.1 \\
\hline
\end{tabular}

Medians (interquartile range) for continuous variables

VAS - Visual Analog Scale, HAQ - Health Assessment Questionnaire, DAS28 - Disease Activity Score 28 joints, CRP - C-reactive protein, RF - Rheumatoid Factor, ACPA - AntiCitrullinated Protein Antibodies.

Table 2. Shared epitope allele carrier frequencies.

\begin{tabular}{llll}
\hline Allele & $\begin{array}{c}\text { Danish patients } \\
\mathrm{n}=98\end{array}$ & $\begin{array}{c}\text { Turkish patients } \\
\mathrm{n}=95\end{array}$ & P-value \\
\hline $\mathrm{S} 1, \%(\mathrm{n})$ & $19(37)$ & $22(42)$ & 0,43 \\
$\mathrm{~S} 2, \%(\mathrm{n})$ & $26(51)$ & $8(16)$ & $<0,00001$ \\
S3D, \% (n) & $6(12)$ & $21(39)$ & 0,000029 \\
S3P, \% (n) & $27(52)$ & $29(56)$ & 0,52 \\
$\mathrm{X}, \%(\mathrm{n})$ & $22(44)$ & $19(37)$ & 0,47 \\
\hline
\end{tabular}

We found no associations between the risk-enhancing alleles and the presence of IgM rheumatoid factor or ACPA.

Conclusion: The Turkish patients were younger and had lower disease activity than Danish at the time of diagnosis. Our study found an enhanced genetic susceptibility to RA in Danish compared to Turkish patients with a higher prevalence of risk-enhancing RA alleles and a lower prevalence of protective alleles. References:

[1] Tezenas du Montcel S, Michou L, Petit-Teixeira E, Osorio J, Lemaire I, Las bleiz $\mathrm{S}$, et al. New classification of HLA-DRB1 alleles supports the shared epitope hypothesis of rheumatoid arthritis susceptibility. Arthritis Rheum 2005; 52: 1063-8.

Disclosure of Interests: None declared

DOI: 10.1136/annrheumdis-2020-eular.3963

\section{AB0203 LUNG DISEASE CHARACTERISTICS IN MOROCCAN RHEUMATOID ARTHRITIS PATIENTS}

B. Laila ${ }^{1}$, H. Azzouzi ${ }^{1}$, I. Linda ${ }^{1} .{ }^{1}$ University Mohammed First Faculty of Medicine, Oujda, Morocco

Background: Pulmonary involvement is the most common extra-articular Rheumatoid Arthritis (RA) manifestation. Interstitial lung disease is an important and early feature and can increase the mortality risk in RA patients. In Morocco no previous studies have been carried out to identify the prevalence of lung disease in RA patients nor have the risk factors for development of interstiel lung disease (ILD)

Objectives: The aim of this study was to investigate the prevalence of lung disease and analyse the ILD associated risk factors, in Moroccan patients with rheumatoid arthritis.

Methods: This was a retrospective analysis of 288 patients diagnosed with RA between January 2014 and December 2019. Exclusion criteria were: pregnant women, history of other autoimmune disease than RA, pulmonary tuberculosis diagnostignosed before lung exploration, any drugs known to cause pulmonary changes (such as Amiodarone). Clinical, and laboratory features were recorded simultaneously with the period of pulmonary exploration. Lung involvement was 